\title{
GSTT1, GSTM1, and GSTP1 polymorphisms as a prognostic factor in women with breast cancer
}

\author{
A.L. Oliveira ${ }^{1}$, F.F. Oliveira Rodrigues ${ }^{1}$, R.E. dos Santos ${ }^{1}$, \\ R.L. Rozenowicz ${ }^{1}$ and M. Barbosa de Melo ${ }^{2}$ \\ ${ }^{1}$ Departamento de Obstetrícia e Ginecologia, \\ Irmandade da Santa Casa de Misericórdia de São Paulo, São Paulo, SP, Brasil \\ ${ }^{2}$ Laboratório de Genética Molecular Humana, \\ Centro de Biologia Molecular e Engenharia Genética, \\ Universidade Estadual de Campinas, Campinas, SP, Brasil \\ Corresponding author: A.L. Oliveira \\ E-mail: drandrel@terra.com.br
}

Genet. Mol. Res. 13 (2): 2521-2530 (2014)

Received September 10, 2013

Accepted November 10, 2013

Published January 22, 2014

DOI http://dx.doi.org/10.4238/2014.January.22.9

\begin{abstract}
The glutathione S-transferase (GST) family comprises phase-II cellular detoxification enzymes that catalyze the conjugation of chemotherapy drugs to glutathione and act on the apoptotic pathway. The aim of this study was to determine whether polymorphisms of the GSTT1, GSTM1, and GSTP1 genes are associated with different rates of overall survival (OS) and disease-free survival (DFS) after neoadjuvant chemotherapy in the management of locally advanced breast cancer, using either simple or combined analyses, and in relation to the posttherapy axillary lymph node status. Forty women with invasive ductal carcinoma of the breast submitted to neoadjuvant chemotherapy with 5-fluorouracil, epirubicin, and cyclophosphamide were genotyped for GSTT1, GSTM1, and GSTP1. Comparisons were performed for the three genes, either isolated or in pairs, in polymorphic or wild-type combinations. Finally, the OS and DFS of patients were analyzed with
\end{abstract}


respect to axillary lymph node status and with respect to wild-type or polymorphic presentations of each gene. No statistically significant difference in OS and DFS was evident between women with wildtype or polymorphic forms of the genes, either isolated or in pairs, after neoadjuvant chemotherapy. By contrast, after treatment, lymph node-negative women had better OS and DFS only in the presence of polymorphisms of GSTP1, and improved DFS only in the presence of the polymorphic types of GSTT1 and GSTM1 compared to women with positive lymph nodes. The presence of polymorphic forms of GSTP1, GSTM1, and GSTT1 was crucial to conferring better OS and DFS among women with negative axillary lymph nodes.

Key words: Breast cancer; Polymorphism; Genetic; Drug therapy; Glutathione transferase

\section{INTRODUCTION}

Breast cancer (BC) is the most prevalent gynecological neoplasia in women worldwide, with an estimated 207,090 new cases and 39,840 deaths in 2010 for the United States alone (Jemal et al., 2010). Since the first studies in solid tumors, the role of adjuvant chemotherapy has become increasingly important in the treatment of locally advanced breast tumors (Shapiro and Fugmann, 1957).

Advanced stages of the disease and drug resistance mechanisms represent the greatest barriers to effective treatment of BC (Leonessa and Clarke, 2003). Consequently, there have been major shifts in the paradigms for managing $\mathrm{BC}$, leading to increasingly multi-disciplinary approaches.

Drawing on knowledge of the cell cycle and mechanisms of action of the chemotherapeutic drugs used in $\mathrm{BC}$, a multi-drug treatment strategy has been adopted that acts at different phases of the cell cycle, conferring greater patient survival and longer disease-free periods after surgical treatment (Fisher et al., 1968). However, evidence also shows that women at the same clinical stage of $\mathrm{BC}$ react differently to the same treatment, with some groups presenting with disease progression. The main reasons for failure of chemotherapy in cancer patients are disease spread and drug-resistance mechanisms (Terek et al., 2003; Jemal et al., 2010). Biochemical and molecular aspects of this resistance process have been described in a number of studies, implicating a significant contribution of enzymes encoded by genes of the glutathione S-transferase family (GSTs) (Hayes and Pulford, 1995; O'Brien and Tew, 1996; Burg and Mulder, 2002; Townsend and Tew, 2003a,b; L'Ecuyer et al., 2004).

Theta class proteins are encoded by two genes (GSTT1 and GSTT2) that are located on chromosome 22. The presence of the GSTT1 null form, which does not produce the enzymes purifying cellular metabolism, has been associated with enhanced responses to chemotherapy in patients with BC (Khedhaier et al., 2003).

Proteins belonging to the Mu class are encoded by a group of genes located on chromosome 1 (GSTM 1-5). The GSTM1 null genotype is unable to produce the catalyzing enzymes necessary for the process of conjugation with glutathione. In addition, individuals with this null genotype do not synthesize the proteins that can bind proteins from the ASK1 pathway, 
which are required for inhibition of this apoptotic pathway (Figure 1). These polymorphic forms are associated with improved responses to some classes of chemotherapeutic drugs that are currently used against a range of cancer types (Ambrosone et al., 2001; Cho et al., 2001; Townsend and Tew, 2003a,b; Khedhaier et al., 2003; McIlwain et al., 2006).

The Pi class of GST proteins comprises only a single protein that is encoded by a gene located on chromosome 11 called GSTP1. With regards to its role in cellular protection, this gene is relatively more associated with the apoptotic process. However, polymorphic forms of GSTP1 are unable to synthesize the proteins that bind to enzymes of the JNK pathway, and therefore cannot inhibit this apoptotic pathway (Adler et al., 1999; Dang et al., 2005) (Figure 1). In addition, these polymorphisms do not encode cellular detoxification enzymes.

Previous studies associating the presence of polymorphic forms of GST genes with responses to cancer chemotherapy have shown conflicting results; moreover, these are generally based on inconsistent data (Riddick et al., 2005). This prompted our group to investigate overall survival (OS) and disease-free survival (DFS) in women with invasive ductal carcinoma of the breast (stages II and III) in relation to the presence of the polymorphisms GSTT1/ GSTT1 "null", GSTM1/GSTM1 "null", and GSTP1 Ile105Ile/ GSTP1 Ile105Val.

\section{MATERIAL AND METHODS}

\section{Patients}

This longitudinal prospective study, which was approved by the Research Ethics Committee of Santa Casa de São Paulo Hospital, involved a randomized sample of 52 women with clinically confirmed breast cancer who were seen at the Department of Obstetrics and Gynecology of Santa Casa de São Paulo Hospital, Brazil. Subjects were recruited between July 2004 and July 2006, and all patients underwent clinical, laboratory, and radiological follow-up up to December 2009. The study inclusion criteria were as follows: patients aged between 30 and 75 years with singular, unilateral, primary neoplasia of the breast (assessed by mammography, ultrasonography, and magnetic resonance imaging of the breast) with UICC stage II or III invasive ductal histology in the absence of cardiopathy.

Of the initial 52 patients recruited, 12 were excluded: one patient was diagnosed with granulomatous mastitis, one patient presented morbid obesity precluding the magnetic resonance imaging examination, three patients had metastatic tumors, six patients were diagnosed with invasive lobular carcinoma, and one patient was excluded because her DNA sample presented degradation. This resulted in a final group of 40 patients, all of whom met the inclusion criteria and signed an informed consent form. Patients were submitted to skin tattooing to mark the clinical limits of the initial tumors prior to starting chemotherapy. Chemotherapy was performed in three preoperative cycles, interspersed by 21-day intervals, using the 5-fluorouracil-epirubicin-cyclophosphamide scheme. Patients were assessed clinically according to the criteria adopted by the Response Evaluation Criteria in Solid Tumors Group (RECIST) (Therasse et al., 2000). Subjects presenting greater than or equal to $30 \%$ reduction in tumor diameter upon clinical measurements were considered responders, whereas those showing a less than $30 \%$ reduction in tumor diameter, or presenting disease stability or progression were classified as non-responders. Upon completing three primary chemotherapy cycles, patients were submitted to operative therapy based on radical or conservative surgery. Follow-up en- 
tailed review of the patients' medical records up to December 2009.

\section{DNA extraction}

A 5-mL venous blood sample was taken from the peripheral antecubital ulnar vein and placed in a sterile vial containing 10\% edetic acid (EDTA) as an anti-coagulant. DNA extraction was performed using the salting-out method proposed by Lahiri and Nurberger (1991) as modified by Cavalli et al. (1996) and Salazar et al. (1998).

\section{Multiplex polymerase chain reaction (PCR) of GSTT1 and GSTM1 and enzymatic digestion PCR of polymorphic fragments of GSTP1}

The genotypes of GSTM1 and GSTT1 were determined by co-amplification using multiplex PCR, with the $\beta$-globin gene as the internal control, as described by Wilson et al. (2000). The products related to the GSTP1 gene were obtained by PCR-restriction fragment length polymorphism (RFLP). The amplified product was then digested with the Alw26I restriction enzyme (New England Biolabs; Ipswich, MA, USA), yielding 91- and 86-bp fragments for the homozygous GSTP1105Val genotype, 176-, 91-, and 86-bp fragments for the heterozygous GSTP1Ile105Val genotype, and a single 176-bp fragment for the wild-type GSTP1105Ile genotype.

\section{Statistical analysis}

Statistical analyses were performed using the Microsoft Excel program version 2007 to create the study database. Kaplan-Meier curves were applied for the determination of OS and DFS and chi-squared (Pearson's or Fisher's exact) tests of significance were employed with a $95 \%$ confidence interval.

\section{RESULTS}

In the present study, the mean and median age of patients assessed was 52 years. Six patients were classified as stage IIa, 22 as stage IIb, and 12 as stage IIIa. Out of the 40 patients studied, five (12.5\%) showed a full pathological response according to RECIST criteria. Anatomopathological analysis revealed that 20 patients were lymph node-positive and 20 patients were lymph node-negative post-surgery; $24(60 \%)$ were considered to be responders and $16(40 \%)$ were non-responders according to RECIST criteria. The mean DFS observed was 43.8 months (range: 4.2-64.4 months) and the mean OS was 50.3 months (range: 19.3-64.6 months). Eleven patients died during the follow-up period due to disease progression.

There was no statistically significant difference in OS and DFS between the wildtype and polymorphic forms of GSTT1, GSTM1, and GSTP1 when analyzed either isolated or paired. Women with negative axilla showed a statistically significant difference in DFS and OS in the comparative analysis of the pathological status of lymph nodes after neoadjuvant chemotherapy. However, women with negative axillary lymph nodes showed significantly increased OS and DFS compared to women with positive axillary lymph nodes only in the presence of polymorphic forms of GSTT1, GSTP1, and GSTM1 (Figures 1 to 6). 


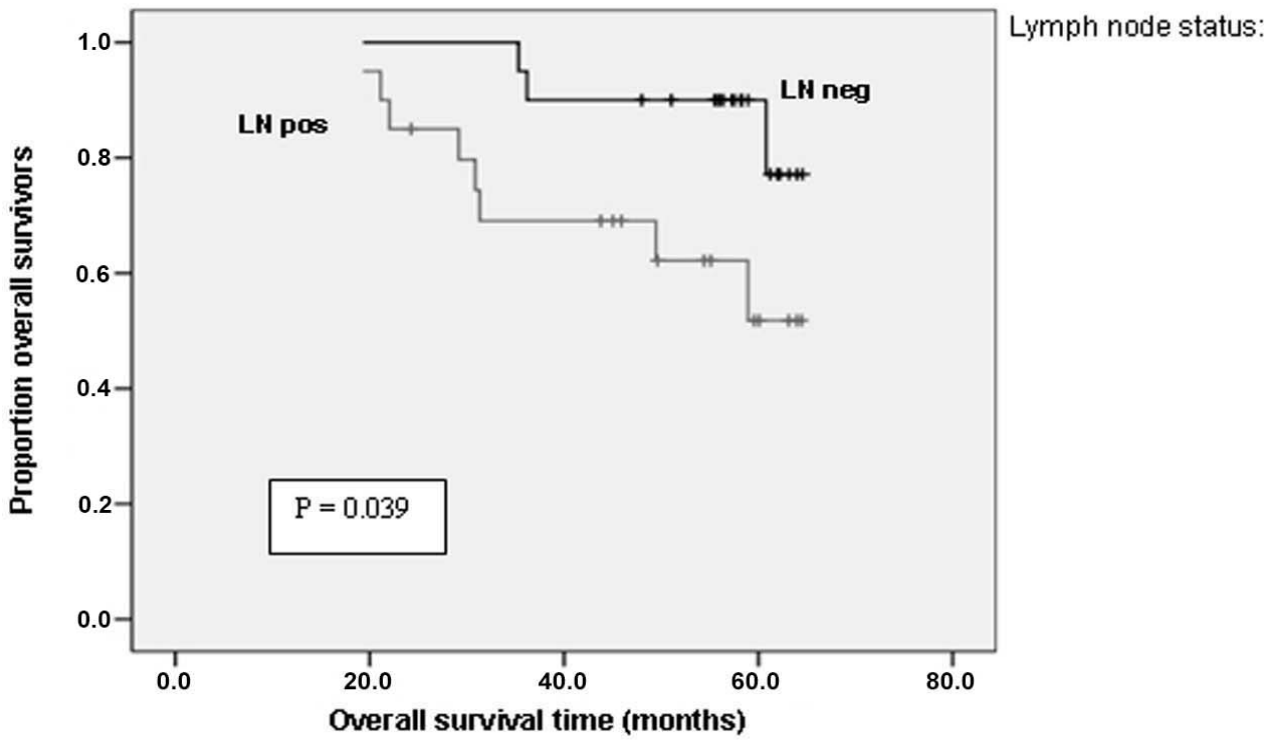

Figure 1. Overall survival in patients with positive or negative lymph nodes after neoadjuvant chemotherapy and surgery.

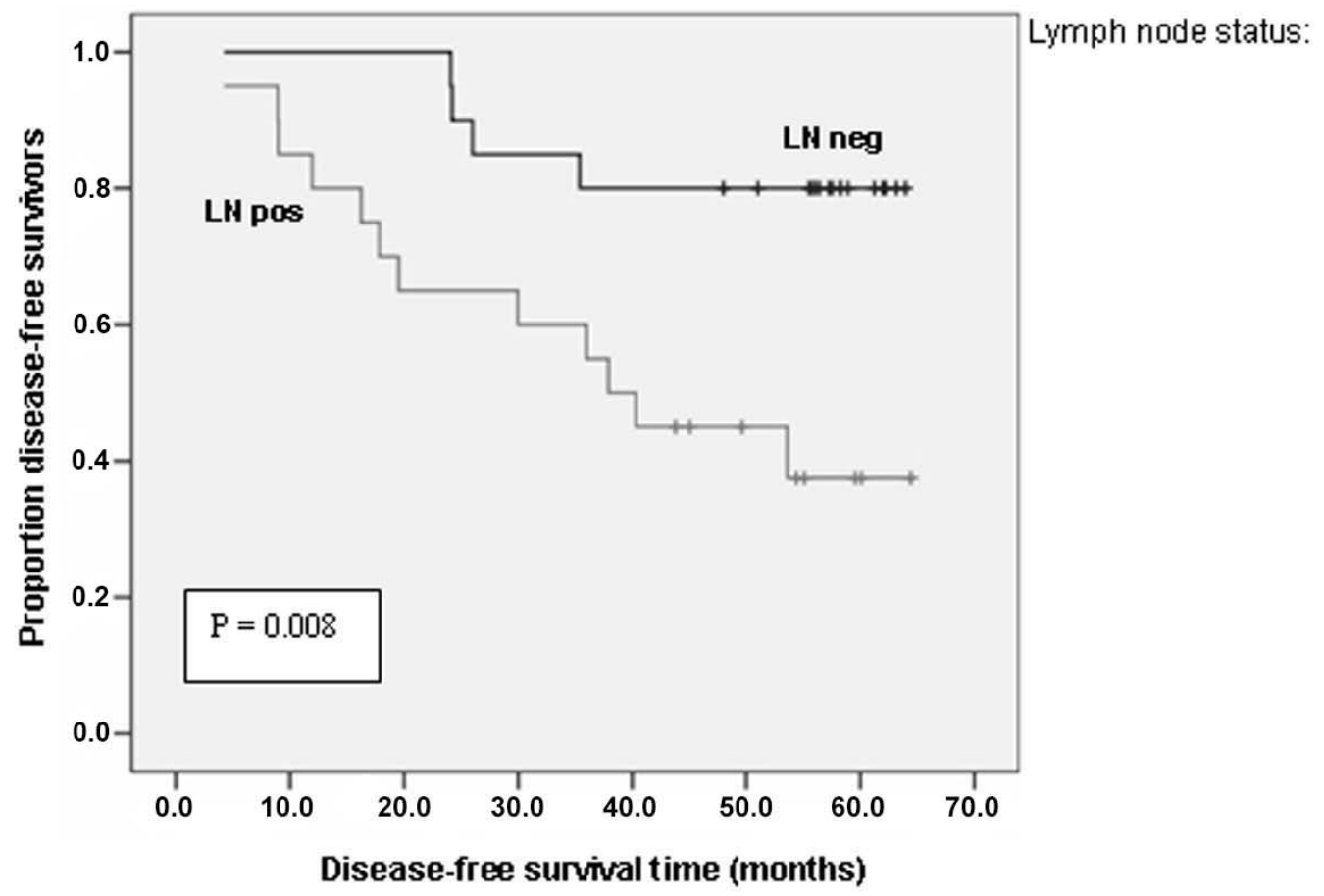

Figure 2. Disease-free survival in patients with positive or negative lymph nodes after neoadjuvant chemotherapy and surgery. 


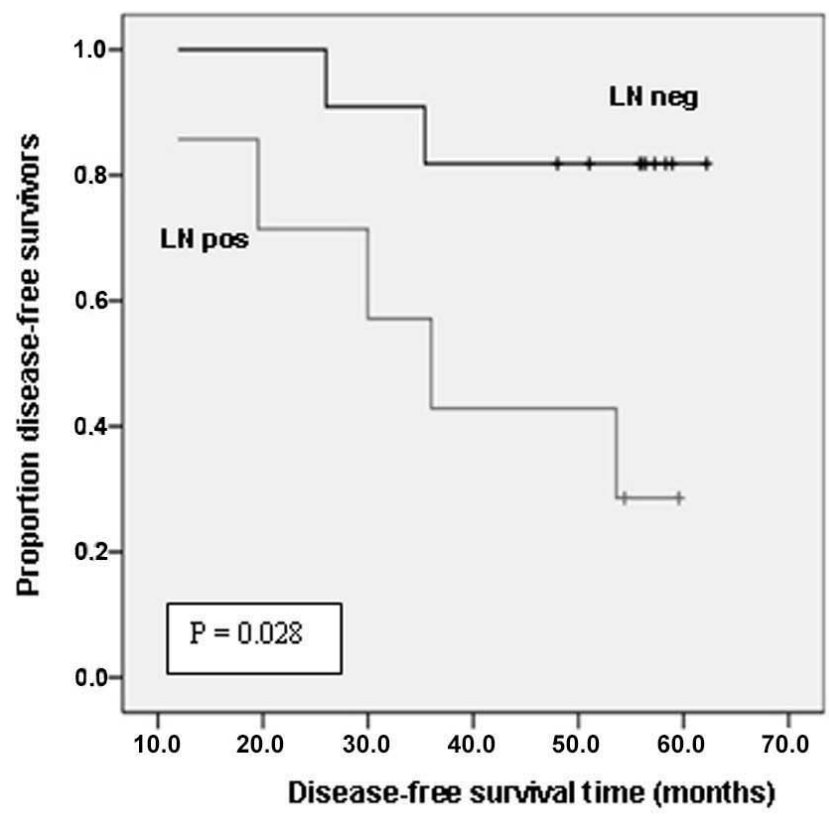

Lymph node status:

Figure 3. Disease-free survival in patients with positive or negative lymph nodes after neoadjuvant chemotherapy and surgery among women with the polymorphic form of the GSTT1 gene.

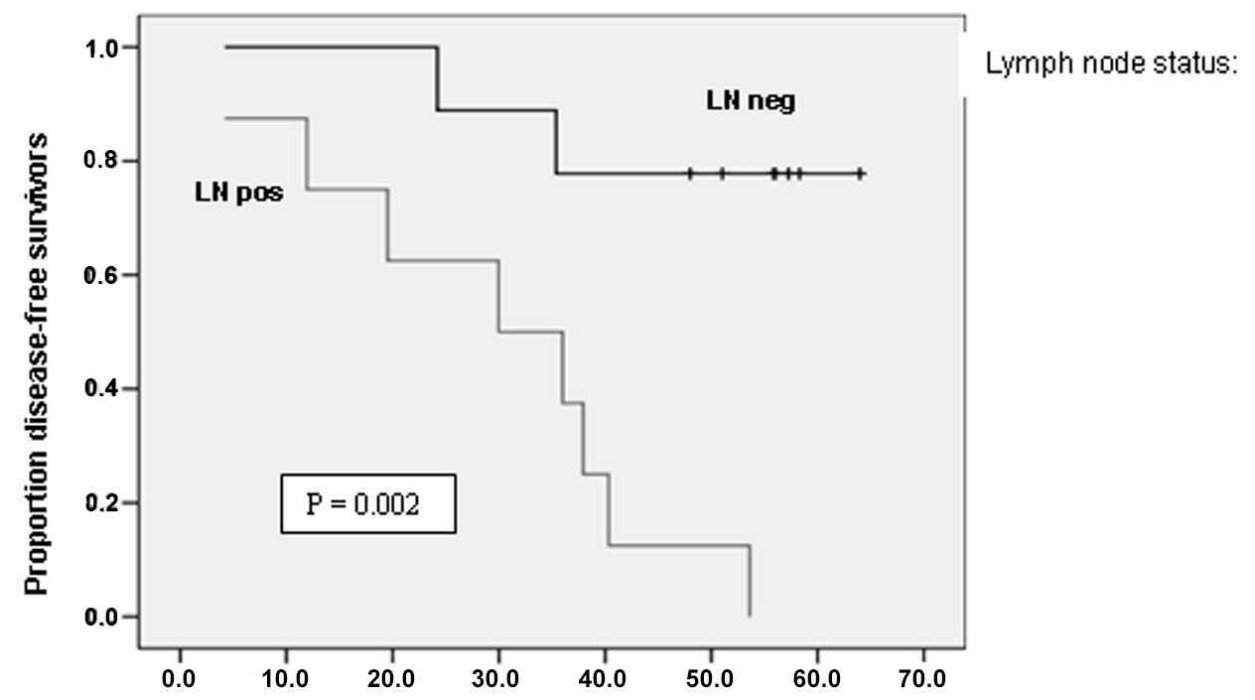

Disease-free survival time (months)

Figure 4. Disease-free survival in patients with positive or negative lymph nodes after neoadjuvant chemotherapy and surgery among women with the polymorphic form of the GSTM1 gene. 


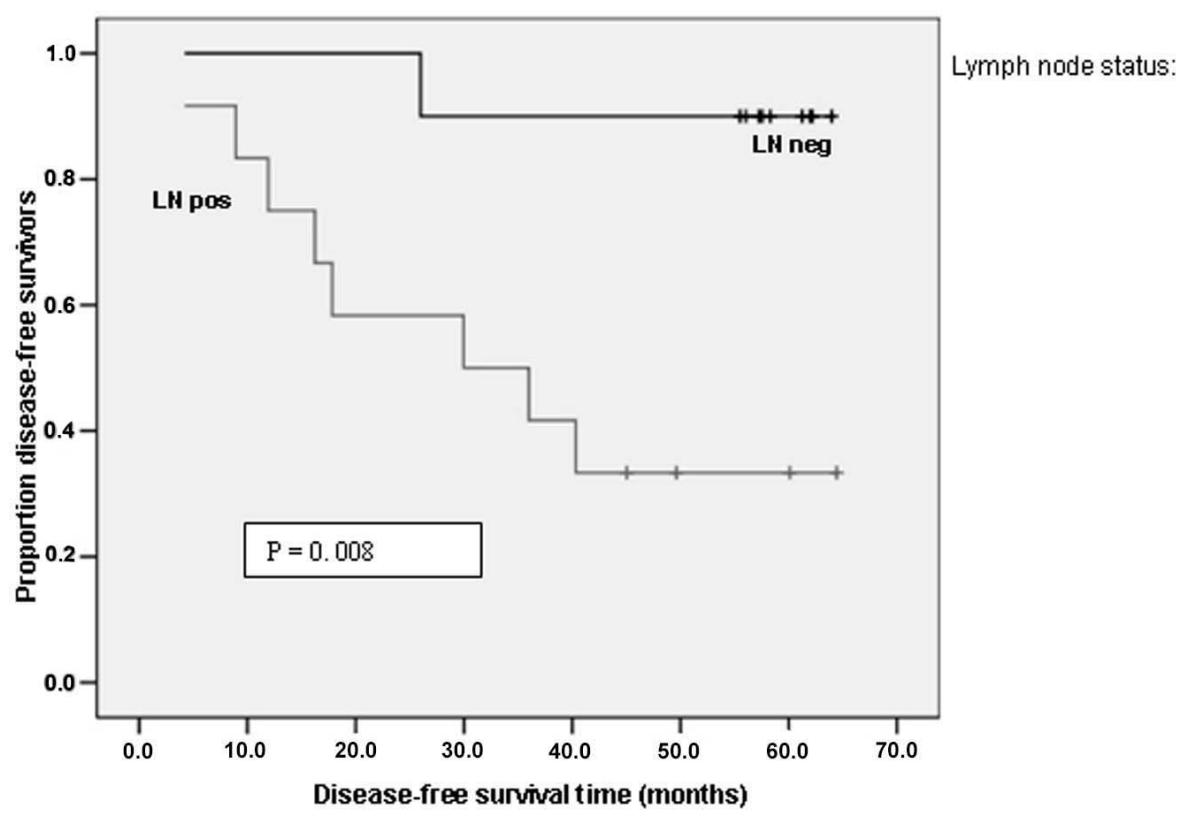

Figure 5. Disease-free survival in patients with positive or negative lymph nodes after neoadjuvant chemotherapy and surgery among women with the polymorphic form of the GSTP1 gene.

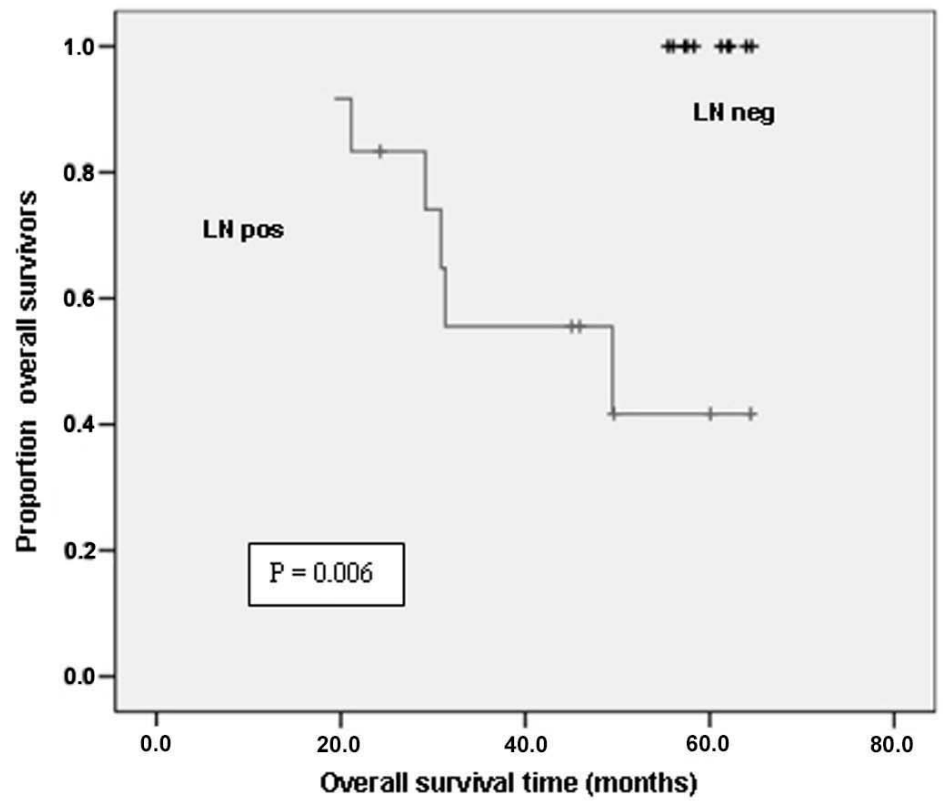

Lymph node status:

Figure 6. Overall survival in patients with positive or negative lymph nodes after neoadjuvant chemotherapy and surgery among women with the polymorphic form of the GSTP1 gene. 


\section{DISCUSSION}

The present study, which examined the three genes from the GST family that show associations with BC (GSTT1, GSTM1, and GSTP1), adopted two specific and unique features compared with previous related studies: i) a longitudinal prospective study design was employed to analyze OS and DFS based on molecular biology; ii) the behavior of these genes was studied in relation to anatomopathological lymph node status as a prognostic factor for OS and DFS.

Numerous studies have sought to establish a relationship between the response to chemotherapy and the action of cellular survival mechanisms, and to ascertain the specific impact of this cellular process on the prognosis of cancer patients. This process is related to the action of proteins that enable the efflux of drugs by cellular detoxification mechanisms and changes in apoptotic pathways, both of which are promoted by the genes in the GST family investigated in the present study.

The NSABP B18 protocol appeared to confirm an absence of any effect of neoadjuvant chemotherapy on OS or DFS. However, further analyses of these patients showed a tendency toward benefits in individuals younger than 50 years. Furthermore, the presence of a full pathological response is a prognostic factor that predicts enhanced rates of survival in patients undergoing neoadjuvant chemotherapy, especially when post-treatment lymph node status reveals the absence of metastases (Fisher et al., 1998; Wolmark et al., 2001; Rastogi et al., 2008).

In the first phase of the study, a full response was observed in five patients $(12.5 \%)$ (Oliveira et al. 2010), whereas here, in the second phase, the absence of lymph node invasion after chemotherapy was found to be a determinant of longer OS and DFS $(\mathrm{P}=0.039$ and $\mathrm{P}=$ 0.008). Besides determining the loco-regional response after neo-adjuvant chemotherapy and isolated survival assessment in lymph node-negative women, another objective of this study was to ascertain whether the mechanism of action of the polymorphic forms of the genes studied exerted an effect on survival among these women when analyzed separately, or whether they correlated with the pathological status of lymph nodes after chemotherapy and surgery.

In our previous studies, the clinical response to neoadjuvant chemotherapy was shown to be independent of the types of polymorphisms of the $M D R-1$ (Rodrigues et al., 2008) gene and GSTT1, GSTM1, and GSTP1 (Oliveira et al., 2010).

Follow-up of this patient cohort showed that the presence of wild-type or polymorphic forms of the GSTT1, GSTM1, and GSTP1 genes, either alone or in pairs, had no impact on OS and DFS. In contrast with our findings, other authors have observed enhanced survival rates among women with BC who are carriers of the polymorphic form of these genes (Sweeney et al., 2000; Ambrosone et al., 2001).

Corroborating the findings of previous reports showing that lymph node-negative women have greater OS and DFS after chemotherapy (Fisher et al., 1998; Wolmark et al., 2001; Rastogi et al., 2008), our data confirmed that the absence of neoplastic invasion of the lymph nodes after chemotherapy was a determinant of greater OS and DFS when analyzed separately $(\mathrm{P}=0.039$ and $\mathrm{P}=0.008)$. However, our results indicated that the significant difference in DFS in node-negative women only occurred in carriers of the polymorphic forms of GSTT1 $(\mathrm{P}=0.028)$ and GSTM1 $(\mathrm{P}=0.002)$, whereas those with the polymorphic form of GSTP1 had both greater DFS $(\mathrm{P}=0.008)$ and OS $(\mathrm{P}=0.006)$. Interestingly, the same improved survival rates were not evident when histopathological assessment of lymph nodes, even when negative, involved wild-type forms of the same genes. 
In line with the present study of BC, previous investigations of Hodgkin's lymphoma, ovarian cancer, colorectal cancer, and even $\mathrm{BC}$ have also found the same improved survival rates when lymph node status was associated with polymorphic forms of GSTT1, GSTM1, and GSTP1 (Khedhaier et al., 2003; Huang et al., 2003; Hohaus et al., 2005; DeMichele et al., 2005; Beeghly et al., 2006; Holley et al., 2006; Kweekel et al., 2008; Kolwijck et al., 2009; Jones et al., 2009; Khrunin et al., 2010).

Based on these results and the concepts outlined, we observed that the presence of the wild-type genotype of GSTM1, GSTT1, and GSTP1 had a negative impact on both DFS and OS in the lymph node-negative patient group. This association indicates that the polymorphic genotypes of these same genes promote a greater sensitivity to chemotherapy, which is most likely due to the differential role of the polymorphic forms of genes from the GST family in the process of apoptosis and cellular detoxification, especially the polymorphic form of GSTP1.

The presence of polymorphic forms of GSTP1, GSTM1, and GSTT1 was crucial to improve OS and DFS among women with negative axillary lymph nodes.

\section{ACKNOWLEDGMENTS}

We are grateful to the Institute of Research of Faculty of Medical Sciences and the Support Center for Scientific Publications of Santa Casa de São Paulo for editorial assistance.

\section{REFERENCES}

Adler V, Yin Z, Fuchs SY, Benezra M, et al. (1999). Regulation of JNK signaling by GSTp. EMBO J. 18: 1321-1334.

Ambrosone CB, Sweeney C, Coles BF, Thompson PA, et al. (2001). Polymorphisms in glutathione S-transferases (GSTM1 and GSTT1) and survival after treatment for breast cancer. Cancer Res. 61: 7130-7135.

Beeghly A, Katsaros D, Chen H, Fracchioli S, et al. (2006). Glutathione S-transferase polymorphisms and ovarian cancer treatment and survival. Gynecol. Oncol. 100: 330-337.

Burg D and Mulder GJ (2002). Glutathione conjugates and their synthetic derivatives as inhibitors of glutathionedependent enzymes involved in cancer and drug resistance. Drug. Metab. Rev. 34: 821-863.

Cavalli SA, Otta MI, Hirata RDC and Nguyen NY (1996). Apolipoprotein E genotyping in Brazilian normocholesterolemic individuals. Clin. Chem. 42: (Suppl): S298.

Cho SG, Lee YH, Park HS, Ryoo K, et al. (2001). Glutathione S-transferase mu modulates the stress-activated signals by suppressing apoptosis signal-regulating kinase 1. J. Biol. Chem. 276: 12749-12755.

Dang DT, Chen F, Kohli M, Rago C, et al. (2005). Glutathione S-transferase pil promotes tumorigenicity in HCT116 human colon cancer cells. Cancer Res. 65: 9485-9494.

DeMichele A, Aplenc R, Botbyl J, Colligan T, et al. (2005). Drug-metabolizing enzyme polymorphisms predict clinical outcome in a node-positive breast cancer cohort. J. Clin. Oncol. 23: 5552-5559.

Fisher B, Ravdin RG, Ausman RK, Slack NH, et al. (1968). Surgical adjuvant chemotherapy in cancer of the breast: results of a decade of cooperative investigation. Ann. Surg. 168: 337-356.

Fisher B, Bryant J, Wolmark N, Mamounas E, et al. (1998). Effect of preoperative chemotherapy on the outcome of women with operable breast cancer. J. Clin. Oncol. 16: 2672-2685.

Hayes JD and Pulford DJ (1995). The glutathione S-transferase supergene family: regulation of GST and the contribution of the isoenzymes to cancer chemoprotection and drug resistance. Crit. Rev. Biochem. Mol. Biol. 30: 445-600.

Hohaus S, Di Ruscio A, Di Febo A, Massini G, et al. (2005). Glutathione S-transferase P1 genotype and prognosis in Hodgkin's lymphoma. Clin. Cancer Res. 11: 2175-2179.

Holley SL, Rajagopal R, Hoban PR, Deakin M, et al. (2006). Polymorphisms in the glutathione S-transferase mu cluster are associated with tumour progression and patient outcome in colorectal cancer. Int. J. Oncol. 28: 231-236.

Huang E, West M and Nevins JR (2003). Gene expression profiling for prediction of clinical characteristics of breast cancer. Recent. Prog. Horm. Res. 58: 55-73.

Jemal A, Siegel R, Xu J and Ward E (2010). Cancer statistics, 2010. CA Cancer J. Clin. 60: 277-300. 
Jones BA, Christensen AR, Wise JP, Sr. and Yu H (2009). Glutathione S-transferase polymorphisms and survival in African-American and white colorectal cancer patients. Cancer Epidemiol. 33: 249-256.

Khedhaier A, Remadi S, Corbex M, Ahmed SB, et al. (2003). Glutathione S-transferases (GSTT1 and GSTM1) gene deletions in Tunisians: susceptibility and prognostic implications in breast carcinoma. Br. J. Cancer 89: 1502-1507.

Khrunin AV, Moisseev A, Gorbunova V and Limborska S (2010). Genetic polymorphisms and the efficacy and toxicity of cisplatin-based chemotherapy in ovarian cancer patients. Pharmacogenomics $J$. 10: 54-61.

Kolwijck E, Zusterzeel PL, Roelofs HM, Hendriks JC, et al. (2009). GSTP1-1 in ovarian cyst fluid and disease outcome of patients with ovarian cancer. Cancer Epidemiol. Biomarkers Prev. 18: 2176-2181.

Kweekel DM, Koopman M, Antonini NF, Van der Straaten T, et al. (2008). GSTP1 Ile105Val polymorphism correlates with progression-free survival in MCRC patients treated with or without irinotecan: a study of the Dutch Colorectal Cancer Group. Br. J. Cancer 99: 1316-1321.

L'Ecuyer T, Allebban Z, Thomas R and Vander HR (2004). Glutathione S-transferase overexpression protects against anthracycline-induced H9C2 cell death. Am. J. Physiol. Heart. Circ. Physiol. 286: H2057-H2064.

Lahiri DK and Nurnberger JI, Jr. (1991). A rapid non-enzymatic method for the preparation of HMW DNA from blood for RFLP studies. Nucleic Acids Res. 19: 5444.

Leonessa F and Clarke R (2003). ATP binding cassette transporters and drug resistance in breast cancer. Endocr. Relat. Cancer 10: 43-73.

McIlwain CC, Townsend DM and Tew KD (2006). Glutathione S-transferase polymorphisms: cancer incidence and therapy. Oncogene 25: 1639-1648.

O’Brien ML and Tew KD (1996). Glutathione and related enzymes in multidrug resistance. Eur. J. Cancer 32A: 967-978.

Oliveira AL, Rodrigues FF, Santos RE, Aoki T, et al. (2010). GSTT1, GSTM1, and GSTP1 polymorphisms and chemotherapy response in locally advanced breast cancer. Genet. Mol. Res. 9: 1045-1053.

Rastogi P, Anderson SJ, Bear HD, Geyer CE, et al. (2008). Preoperative chemotherapy: updates of National Surgical Adjuvant Breast and Bowel Project Protocols B-18 and B-27. J. Clin. Oncol. 26: 778-785.

Riddick DS, Lee C, Ramji S, Chinje EC, et al. (2005). Cancer chemotherapy and drug metabolism. Drug Metab. Dispos. 33: 1083-1096.

Rodrigues FF, Santos RE, Melo MB, Silva MA, et al. (2008). Correlation of polymorphism C3435T of the MDR-1 gene and the response of primary chemotherapy in women with locally advanced breast cancer. Genet. Mol. Res. 7: 177183.

Salazar LA, Hirata MH, Cavalli SA, Machado MO, et al. (1998). Optimized procedure for DNA isolation from fresh and cryopreserved clotted human blood useful in clinical molecular testing. Clin. Chem. 44: 1748-1750.

Shapiro DM and Fugmann RA (1957). A role for chemotherapy as an adjunct to surgery. Cancer Res. 17: 1098-1101.

Sweeney C, McClure GY, Fares MY, Stone A, et al. (2000). Association between survival after treatment for breast cancer and glutathione S-transferase P1 Ile105Val polymorphism. Cancer Res. 60: 5621-5624.

Terek MC, Zekioglu O, Sendag F, Akercan F, et al. (2003). MDR1 gene expression in endometrial carcinoma. Int. J. Gynecol. Cancer 13: 673-677.

Therasse P, Arbuck SG, Eisenhauer EA, Wanders J, et al. (2000). New guidelines to evaluate the response to treatment in solid tumors. European Organization for Research and Treatment of Cancer, National Cancer Institute of the United States, National Cancer Institute of Canada. J. Natl. Cancer Inst. 92:205-216.

Townsend D and Tew K (2003a). Cancer drugs, genetic variation and the glutathione-S-transferase gene family. Am. J. Pharmacogenomics 3: 157-172.

Townsend DM and Tew KD (2003b). The role of glutathione-S-transferase in anti-cancer drug resistance. Oncogene 22: $7369-7375$.

Wilson MH, Grant PJ, Hardie LJ and Wild CP (2000). Glutathione S-transferase M1 null genotype is associated with a decreased risk of myocardial infarction. FASEB J. 14: 791-796.

Wolmark N, Wang J, Mamounas E, Bryant J, et al. (2001). Preoperative chemotherapy in patients with operable breast cancer: nine-year results from National Surgical Adjuvant Breast and Bowel Project B-18. J. Natl. Cancer Inst. Monogr. 96-102. 